\title{
NUMERICAL MODEL OF CURRENT AND SEDIMENT TRANSPORT IN THE WAVE BOUNDARY LAYER
}

\author{
Yuliang Zhu' ${ }^{1}$ Jing $\mathrm{Ma}^{2}$ and Hao Wang ${ }^{2}$
}

\begin{abstract}
Mathematical model is one of the means to study of turbulent wave boundary layer. The paper analysis of the existing model, adopt a more reasonable boundary condition to establish a improved mathematical model of 1DV turbulent wave boundary layer using $\mathrm{k}-\varepsilon$ model. The paper recommends brief flow simulation and mainly introduced the simulation of the sediment concentration. The paper use the eddy-viscosity value which calculation by the mathematical model and the model of You Zaijin on time-invariant eddy-viscosity into the relationship about sediment diffusion coefficient and eddy-viscosity to calculate the sediment concentration. The calculation results turns out the way that use the eddy-viscosity value which calculation by the mathematical model into the relationship can obtain better timely sediment concentration value. When use the model simulates the time-invariant sediment concentration, the two ways have not many distinctions. It means the way that that use the eddy-viscosity value which calculation by the mathematical model into the relationship is feasible.
\end{abstract}

Keywords: turbulent boundary layer; mathematical model; $k$-E model; eddy-viscosity

\section{INTRODUCTION}

It is generally turbulent boundary layer in the coastal region of the shallow water wave boundary. The wave energy and sediment movement of the shallow sea is closely related with this layer (Kang Suha 1995). Under the waves, especially for the pink sand, fine sand, its sediment mainly containing Pro at the end of high sand waves along the direction of sediment transport as the main way. The sediment concentration of the layer at the bottom is especially high, called sheet-flow (A. A. Al-Salem 1993). It is generally believed that under the boundary layer near the bottom contains large quantities of sediment under the water waves (Yan Bin 2006). Qian Ning 1983 pointed out that the wave of sediment movement under the waves mainly in the vicinity of bed (Yang Yihang 1988). We can see the results from Flume experiment at home and abroad, the height of the sediment concentration of the layer is only a few centimeters(Less than $10 \mathrm{~cm}$, and the sheet-flow only a few millimeters, the numerical value correspond to the value from the experiments of wave turbulent boundary layer. In high above the sediment layer is clear water, with little sediment.

Therefore under the conditions of the wave (with the existence of sheet-flow) sediment transport can be considered as the same with the sediment transport of wave boundary layer. The sediment transport under the wave effected by the bed at the bottom, sediment ripple in the wave conditions and the situation in a more moderate when the wave action has become severe, sediment ripple will disappear, and become more flat in bed appears on the sheet-flow. Therefore, the high near-bottom layer in near-shore sediment transport is very important.

Mathematical model of wave boundary layer calculation is generally based on turbulence theory. The mathematical model considering the combined effects of wave and water flow and the model considering water wave only equation is consistent, and the model under waves is relatively easy. In this paper, an analysis of the existing model which is based on the establishment of an improved k- $\varepsilon$ model using the mathematical model of wave turbulent boundary layer, the primary function of the model can simulate the level of the wave boundary layer velocity distribution, the rate of sediment, to obtain the velocity of the wave boundary layer and the law of sediment transport.

\section{MODEL}

\section{Basic Equations}

In this paper, the vertical one-dimensional model of the basic flow equation is a total of four, the basic equation of sediment, that is, from Reynolds equation is transformed to the one-dimensional turbulent boundary layer at the bottom of the vertical velocity wave equation.

$$
\frac{\partial u}{\partial t}=-\frac{1}{\rho} \frac{\partial p}{\partial x}+v_{m} \frac{\partial^{2} u}{\partial z^{2}}+\frac{\partial\left(-\overline{u^{\prime} v^{\prime}}\right)}{\partial z}
$$

\footnotetext{
${ }^{1}$ Key Laboratory of Coastal Disaster and Defence, Hohai University, 1 Xikang Road, Nanjing, 210098, China

${ }^{2}$ Department of Harbour and Waterway Engineering, Hohai University, 1 Xikang Road, Nanjing, 210098, China
} 
It is take boussinesq equation $-\overline{u^{\prime} v^{\prime}}=v_{t} \frac{\partial u}{\partial z}$ and the relationship established in the wave boundary layer (Yang Yihang 1988) $\frac{\partial p}{\partial x}=-\rho \frac{\partial u_{0}}{\partial t}$ into the equation (1), so it is changes to:

$$
\frac{\partial u}{\partial t}=\frac{\partial u_{0}}{\partial t}+\frac{\partial}{\partial z}\left(\beta \frac{\partial u}{\partial z}\right)
$$

For the equation $\beta=v_{m}+v_{t}, v_{m}$ is the movement viscosity, $v_{t}$ is the eddy viscosity coefficient. Followed by simplification to a vertical one-dimensional equations of $\mathrm{k}$ and $\varepsilon$ equations (Wang Deguan 1989)

$$
\begin{aligned}
& \frac{\partial k}{\partial t}=\frac{\partial}{\partial z}\left[\left(v_{m}+\frac{v_{t}}{\sigma_{k}}\right) \frac{\partial k}{\partial z}\right]+v_{t}\left(\frac{\partial u}{\partial z}\right)^{2}-\varepsilon \\
& \frac{\partial \varepsilon}{\partial t}=\frac{\partial}{\partial z}\left[\left(v_{m}+\frac{v_{t}}{\sigma_{\varepsilon}}\right) \frac{\partial \varepsilon}{\partial z}\right]+C_{1 \varepsilon} \frac{\varepsilon}{k} v_{t}\left(\frac{\partial u}{\partial z}\right)^{2}-C_{2 \varepsilon} \frac{\varepsilon^{2}}{k}
\end{aligned}
$$

The equation with the eddy viscosity coefficient

$$
v_{t}=C_{\mu} \frac{k^{2}}{\varepsilon}
$$

The equation (2) (5) describe the movement of the wave turbulent boundary layer. One dimensional convection-diffusion equation is given by:

$$
\frac{\partial c}{\partial t}=w_{s} \frac{\partial c}{\partial z}+\frac{\partial}{\partial z}\left(\varepsilon_{s} \frac{\partial c}{\partial z}\right)
$$

\section{Computational Methods}

The Simplified equations of one-dimensional turbulent boundary layer at the bottom of the vertical velocity wave equation, $\mathrm{k}$ and $\varepsilon$ equations are the form of one-dimensional diffusion equation, The advection of the use of central difference scheme, the use of the whole discrete implicit scheme, In order to guarantee the Stability of numerical calculation, firstly, the positive value of $\mathrm{k}, \varepsilon$ must be ensured, negative value is not allowed even if in the process of calculation. In order to ensure the value of $k 、 \varepsilon$ is always positive, some special treatment must be done on the source term. That is to use the linearization method to deal with the negative slope (Jin Zhongqin 1989). Each equation in a fixed time forms tridiagonal equations and solve the equations with the TDMA method respectively. Equation (5) is the bond of connection between velocity equation and two transport equations in the calculations.

One dimensional convection-diffusion equation (6) has convection item, resulting in the famous "center differential problems". Using central difference leads to the divergence of tridiagonal linear system because it can not be guaranteed strictly diagonally dominant, which need to use a special discretization scheme. To solve this problem, we generally using upwind discretization scheme, the index format, mixed format or discrete power function format. Considered better results, as well as the size of computation, the complexity of the format, choose the format of discrete power function (Tao Wenquan 2001) .

\section{The initial conditions and boundary conditions}

For the velocity, the model we used in this article at the bottom boundary condition is non-slip boundary condition. The up boundary conditions for second-order Stokes wave forms, that is,

$$
U_{0}=u_{1} \cos (\omega t)+u_{2} \cos (2 \omega t)
$$

For the equation, $\omega$ is circular frequency, the reason why this boundary conditions we take is because the offshore waves are generally second-order wave (Zhao Chongiiu 1993), and we can expand the scope of application, but for $\mathrm{k}$ and $\varepsilon$, near the wall boundary conditions normally be admitted to

$$
k=\frac{u_{*}^{2}}{\sqrt{c_{\mu}}}
$$




$$
\varepsilon=\frac{u_{*}^{3}}{\kappa z}
$$

$\kappa$ is karman constant. The upper boundary condition : $z=\delta ; \frac{\partial k}{\partial z}=0, \frac{\partial \varepsilon}{\partial z}=0 . \delta$ is the wave boundary layer thickness. Considering $v_{t}$, substitution of (8) and (9) into (5) at the bottom boundary gives

$$
v_{t}=\kappa u_{*} z
$$

The upper boundary condition is calculated by equation (5). The upper boundary condition of sediment concentration depends on the condition that the sections' vertical flux is zero, that is

$$
w_{s} c+\varepsilon_{s} \frac{\partial c}{\partial z}=0
$$

To give the bottom boundary condition of sediment concentration is just to determine the closeto-bed reference sediment concentration in fact. In this paper, we use the curve relation between shields parameter and the close-to-bed sediment concentration proposed by Fredsoe (1992). Velocity uses cold start and $\mathrm{k} 、 \varepsilon, \mathrm{v}_{\mathrm{t}}$ begin calculation with initial value of positive number.

\section{Grid and Parameter}

Given that the velocity gradient is larger in the lower and smaller in the upper, we adopt nonuniform grid which is dense in the lower and sparse in the upper in a common boundary layer velocity model at the bottom of waves. If you want to continue to build sediment model on the basis of current model, the gradient changes of eddy viscosity coefficient need to be considered. According to the research results of Kajiura (1992)、You Zaijin (1992) and so on, such grid layout is suitable for eddy viscosity coefficient too. We use staggered grid, and we place the eddy viscosity coefficient $v_{t}$ and $k$ 、 $\varepsilon$ in the center location between two velocity value. The upper boundary node of $\mathrm{v}_{\mathrm{t}}$ and $\mathrm{k} 、 \varepsilon$ is arranged outside the upper boundary node of velocity. The number of nodes of $u_{v} v_{t}$ and $k 、 \varepsilon$ are the same.

The grid arrangement of Sediment concentration is consistent with that of velocity, and the grid bottom begins from the height of reference point. So except the difference of the bottom boundary from velocity, the other grids (except the first grid) can be almost the same and sediment concentration nodes have the same location with velocity nodes. Sediment diffusion coefficient is placed in the center of grid, which is the location of eddy viscosity coefficient. But compared to the nodes of eddy viscosity coefficient, the first grid (the bottom boundary node of eddy viscosity coefficient) and the last grid (the upper boundary node of eddy viscosity coefficient ) are abandoned.

The reference height of close-to-bed reference sediment concentration is several times of median diameter of sediment. We take sediment settling velocity $\mathrm{w}_{\mathrm{s}}$ as a constant when calculating, and sediment vertical diffusion coefficient $\varepsilon_{s}$ is given by building linear relationship with eddy viscosity coefficient:

$$
\varepsilon_{s}=\lambda v_{t}
$$

\section{ANALYSIS AND CALCULATION RESULTS}

\section{Verification of current calculation results}

The model uses the experimental data published for verification. The data is mainly from the published thesis of Ribberink and Al-Salem (1995) and oscillation trough experiment carried by Van Doorn (1982). The wave period of the former data is large, and its limitation is lack of velocity measuring point close-to-bed. Though the wave period of the latter data is a little smaller, there is velocity data close-to-bed $(\mathrm{z}<1 \mathrm{~cm})$, making up for the disadvantage of the former data. A combination of both is more appropriate for verification information. Verification results are given in Figure 1. and Figure 2.

The verification results show that the model is useful for calculating velocity. When considering the velocity calculation in the boundary layer, it is difficult to decide the five empirical constants of k- $\varepsilon$ model, so we add the influence of integrated adjustment coefficient $P$ and the varieties of computational domain, with details in literature (Yuliang Zhu 2009). 


\section{Analysis and sediment calculation results}

After the sediment model has been built, we calculate sediment concentration and use the data from Ribberink and Al-Salem (1995) to verify it. The calculation is divided into two parts, one part is about sediment concentration varieties in a whole wave period at a certain height, which is instantaneous sediment concentration verification; the other one part is about the curve which expresses how the average sediment concentration in one wave period changes with height (vertical distribution of timeaveraged sediment concentration). Verification is carried out under two conditions. One method is to substitute instantaneous eddy viscosity coefficient obtained from velocity calculation into equation (12) to calculate, the other one is to substitute time invariant eddy viscosity coefficient model into equation (12) to calculate. To make it simple, $\lambda$ in this paper is set as a constant more than 1.

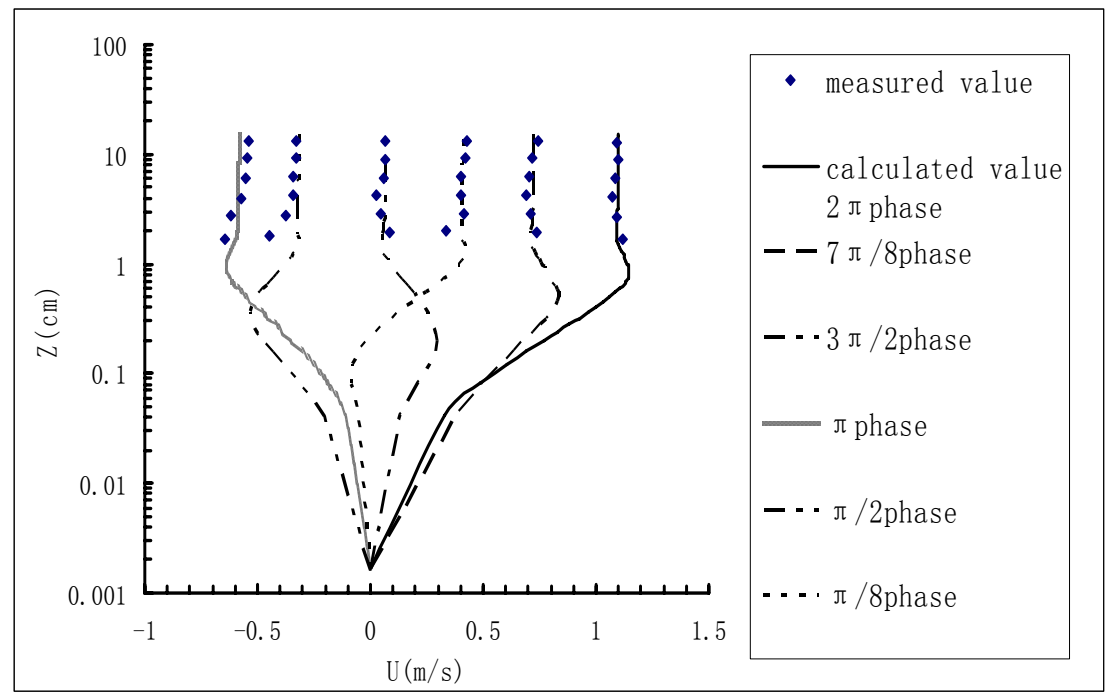

Figure 1. Velocity verification figure of data from Ribberink and Al-Salem

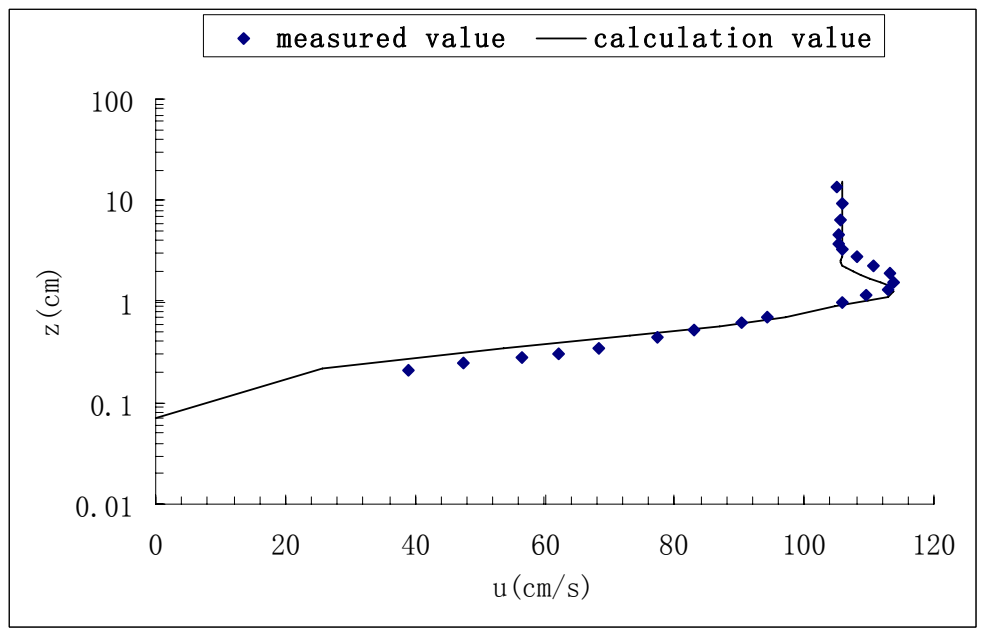

Figure 2. Velocity verification figure of data from Van Doorn 

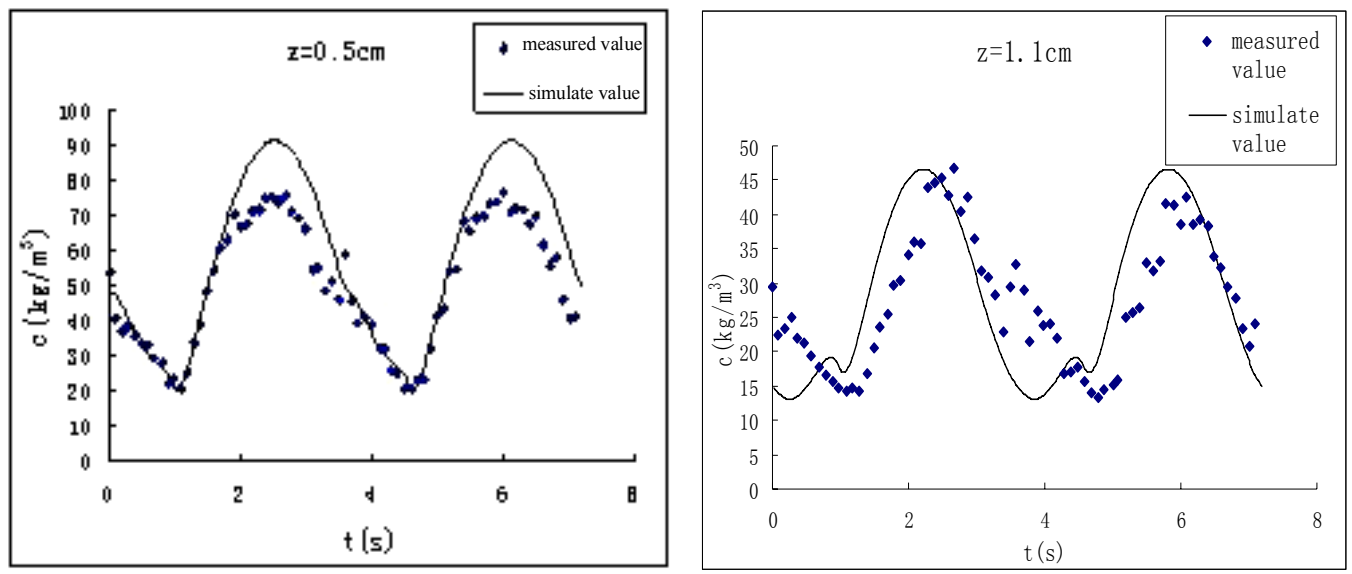

Figure 3a. The sediment concentration at the depth of $0.5 \mathrm{~cm}$ and $1.1 \mathrm{~cm}$ using transient eddy viscositycoefficient
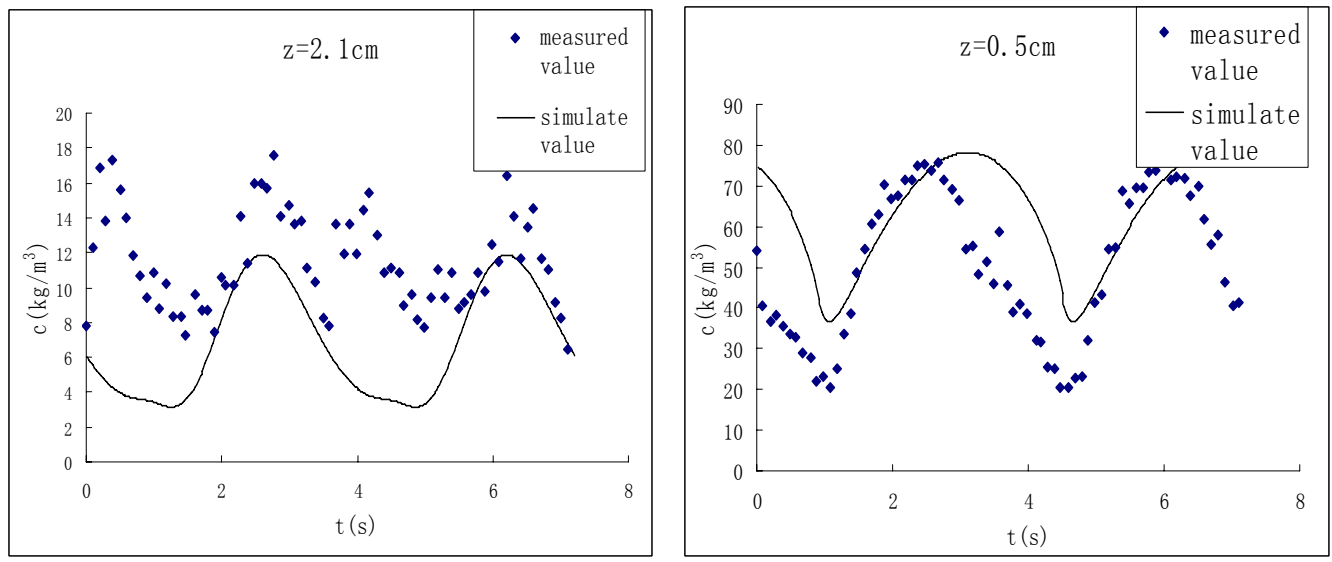

Figure $3 \mathrm{~b}$. The sediment concentration at the depth of Figure 4a. The sediment concentration at the depth $2.1 \mathrm{~cm}$ using transient eddy viscosity-coefficient of $0.5 \mathrm{~cm}$ using You Zaijin model
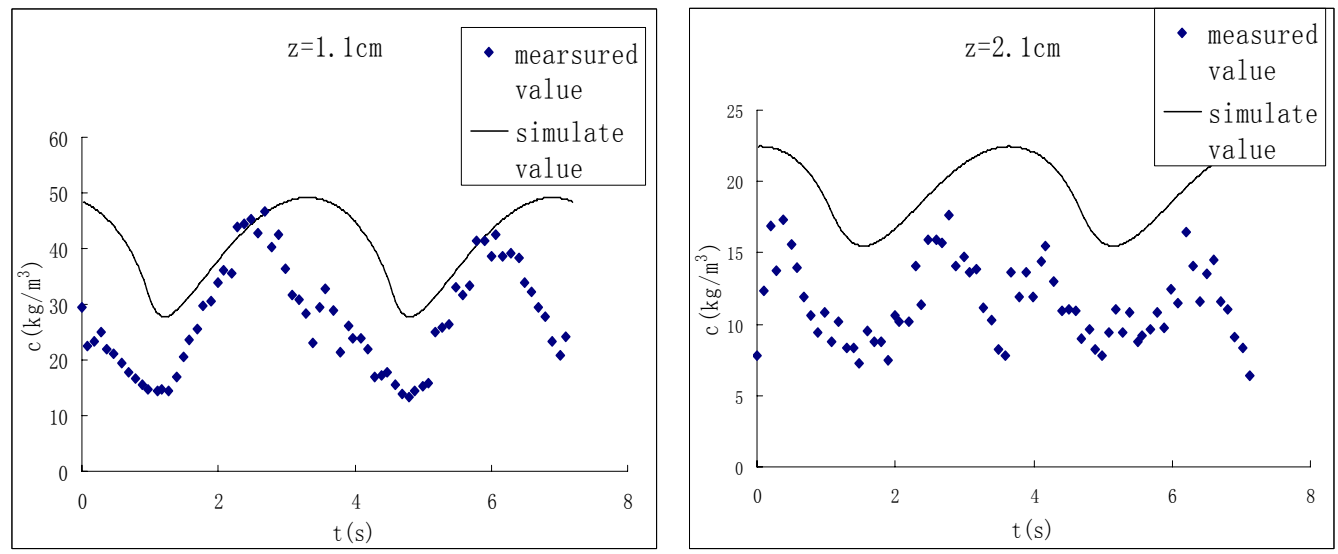

Figure $4 \mathrm{~b}$. The sediment concentration at the depth of $1.1 \mathrm{~cm}$ and $2.1 \mathrm{~cm}$ using You Zaijin model

There are two numerical examples about instantaneous sediment concentration. The basic parameter of Example 1 is wave period $T 7.2 \mathrm{~s}$, sediment median diameter $\mathrm{d}_{50} 0.2 \mathrm{~mm}$ and bottom velocity amplitude of free flow layer $u_{m} 1.7 \mathrm{~m} / \mathrm{s}$. That is symmetric oscillatory flow condition (sine wave). $\mathrm{U}_{0}$ takes $-90^{\circ} \sim 270^{\circ}$ as a wave cycle to make the phase in accord with that of measured data. We compare the numerical simulation results at $\mathrm{z}=0.5 \mathrm{~cm}, 1.1 \mathrm{~cm}$ and $2.1 \mathrm{~cm}$ with the measured data. The calculation results of the two methods are given in Figure 3. and Figure 4. 
From Figure 3. we can see that in the $\mathrm{z}=0.5 \mathrm{~cm}, 1.1 \mathrm{~cm}$ Department, the simulation results in line with the measured quite good. In $\mathrm{z}=2.1 \mathrm{~cm}$ Department the results simulates the basic trend, but the values are smaller. Overall, the simulation results are good. However, when calculating time-invariant eddy viscosity coefficient of the model such as You Zaijin model into the formula (12), some simulation results are worse, mainly manifested as some of the small value of sediment concentration are higher than the measured value of the results during a wave cycle. The results in $\mathrm{Z}=2.1 \mathrm{~cm}$ are a littler bit high. The approach using $k-\varepsilon$ model to calculate the instantaneous coefficient of eddy viscosity and re-build the relationship between the diffusion coefficient shows better applicability than the method using time-invariant eddy viscosity coefficient model such as You Zaijin model ect. With the increase in $\mathrm{z}$, the simulation results of two methods respectively have the problem of too small and too big. We could change $\lambda$ from the constant to function in order to make improvement. Qualitative terms, the former way to increases in the $z$ value of $\lambda$ should be increased, and the latter way is to increases in the $z$ value of $\lambda$ should be appropriately reduced.

Sample 2 is stocks waves, parameters are follows: wave period $\mathrm{T}$ is $6.5 \mathrm{~s}$, the value of sediment particle size $d_{50}$ is $0.2 \mathrm{~mm}$, the free flow speed at the bottom layer amplitude um is $1.1 \mathrm{~m} / \mathrm{s}$, non-wave symmetry $\mathrm{R}=0.66$. There are four measured results for contrast in four heights which are $\mathrm{z}=0.1 \mathrm{~cm}$, $0.5 \mathrm{~cm}, 0.7 \mathrm{~cm}$ and $1.8 \mathrm{~cm}$. The results are showed in Figure 5. and Figure 6. Compare the measured data, we can see that the measured rate of the largest sediment concentration phase shift back as the distance from the bed increasing. The calculated simulation value simulated the trend too, but the back speed is not as large as the measured value. During a wave cycle, sediment concentration of relatively large value of simulation has reached a good result, but simulation of the small value is general. In Figure 5., when the corresponding flow speed is small, the measured value is also small, but there is a small calculated value of the peak, while in Figure 5a., whether measured value or calculated value, there are a small peak. In Figure $5 \mathrm{~b} .(\mathrm{z}=1.8 \mathrm{~cm})$, the measured values and calculated values of the second peak is not obvious.

As the same, calculate time-invariant eddy viscosity coefficient of the model such as You Zaijin model into the formula (12), the results of Figure 6. We can see that, as same as Sample 1, the simulating effect is poor, which mainly manifested as some of the small value of sediment concentration is higher than the measured value of the results during a wave cycle. Compared with sample 1, it has less reference value. The reason why this would happen, in my suspect is that when using sediment diffusive convection equation for calculation, the diffusion coefficient is set to a fixed value, so the time when small sediment concentration corresponds to the small diffusion coefficient too. Using the instantaneous coefficient of eddy viscosity into the formula (12) calculation method (Figure 5.), although there is still this problem, from the calculation results when $z=1.8 \mathrm{~cm}$ we can clearly see that the corresponding wave velocity cycle direction of wave propagation in the opposite direction to the time of the peak sediment concentration tend to disappear, and start to close to the actual situation.
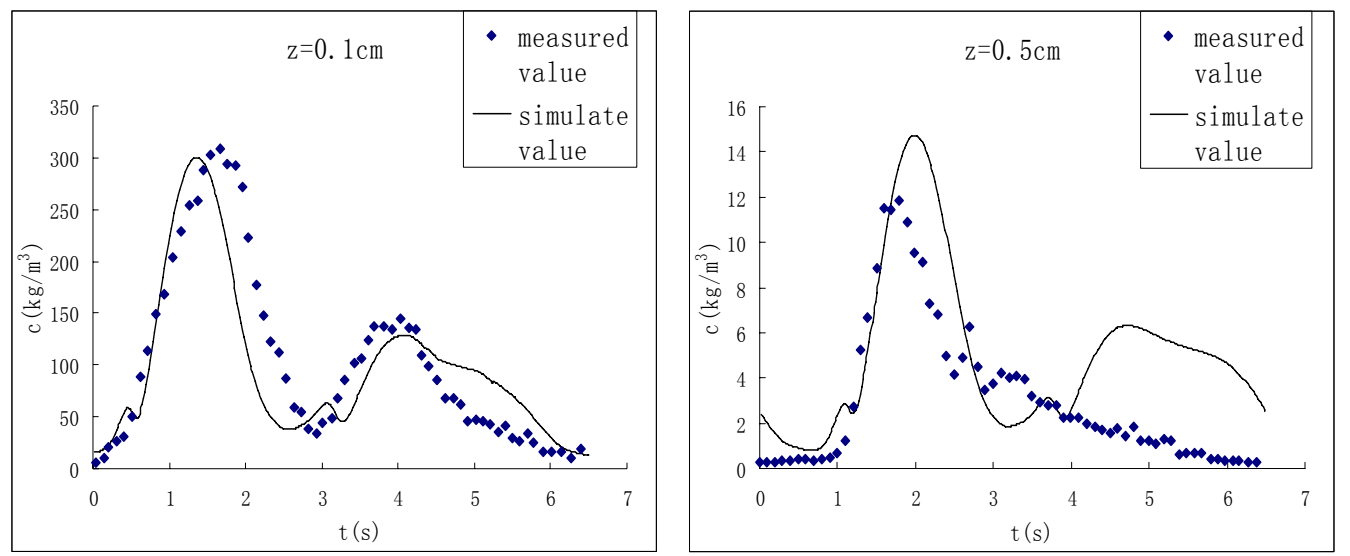

Figure $5 \mathrm{a}$. The sediment concentration at the depth of $0.1 \mathrm{~cm}$ and $0.5 \mathrm{~cm}$ using transient eddy viscositycoefficient 

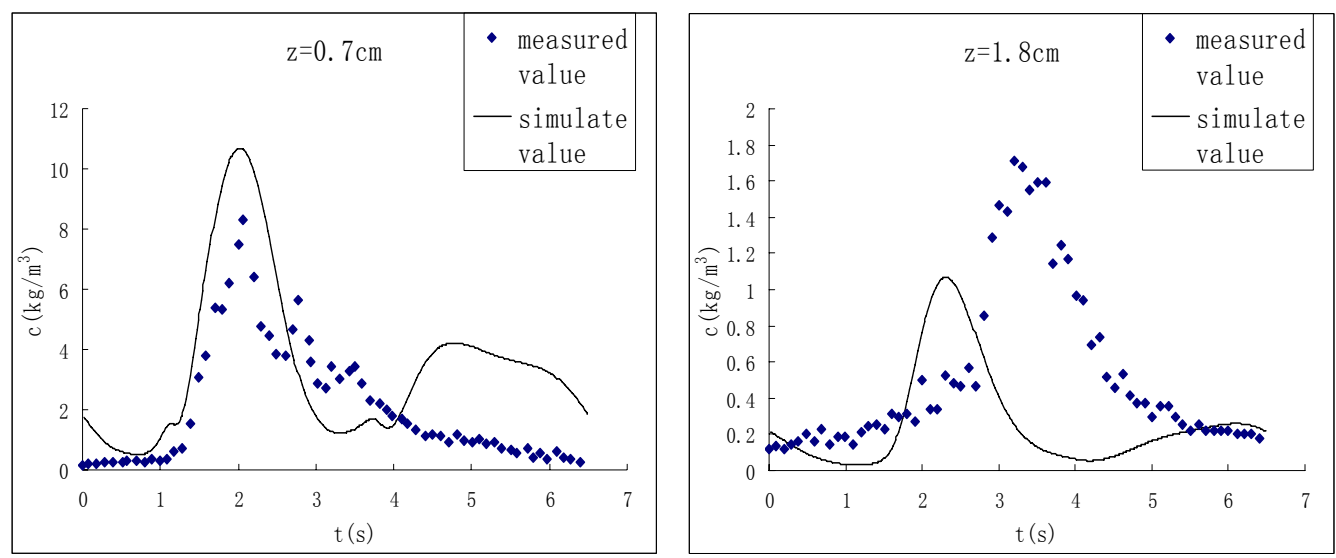

Figure $5 \mathrm{~b}$. The sediment concentration at the depth of $0.7 \mathrm{~cm}$ and $1.8 \mathrm{~cm}$ using transient eddy viscositycoefficient
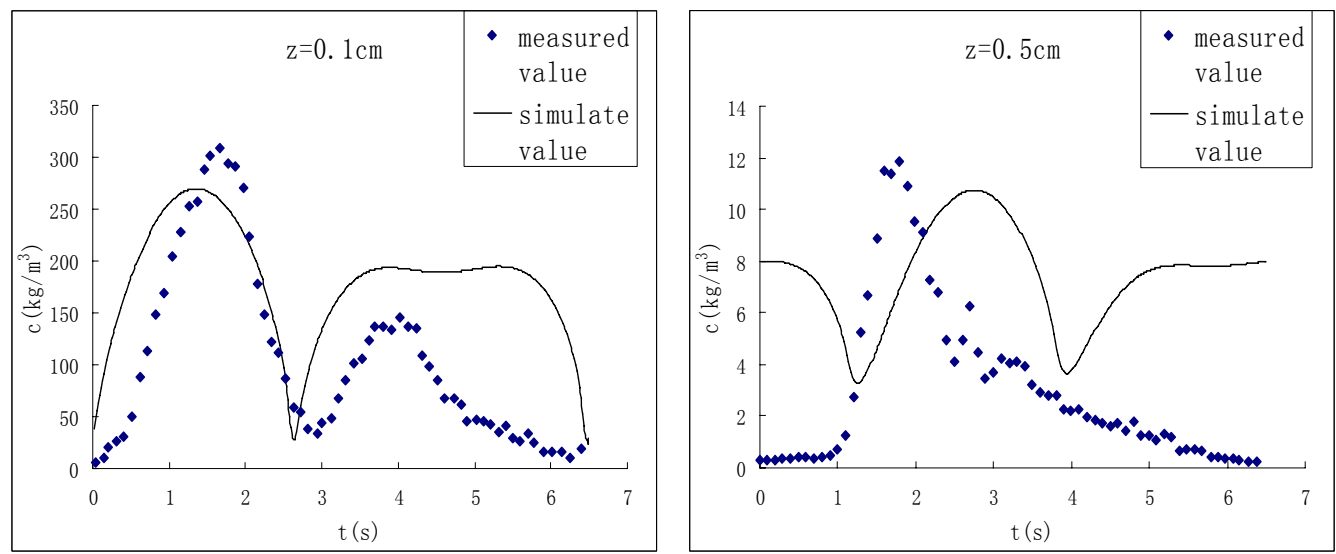

Figure $6 \mathrm{a}$. The sediment concentration at the depth of $0.1 \mathrm{~cm}$ and $0.5 \mathrm{~cm}$ using You Zaijin model
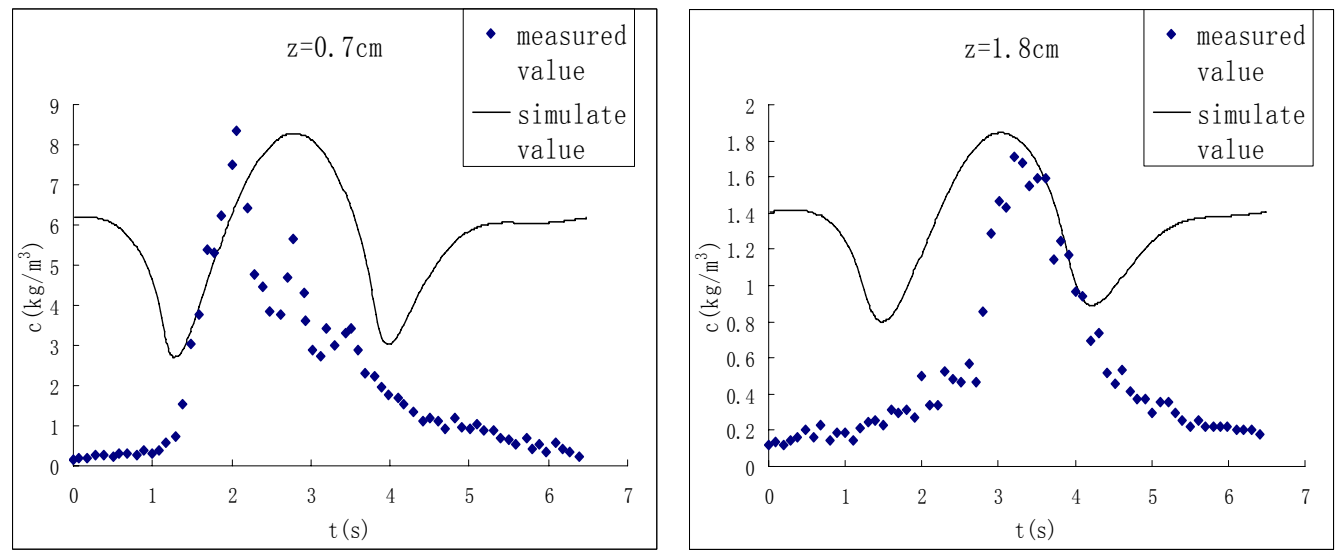

Figure $6 \mathrm{~b}$. The sediment concentration at the depth of $0.7 \mathrm{~cm}$ and $1.8 \mathrm{~cm}$ using You Zaijin model

In the two samples, under the condition of sine wave the measured value of the instantaneous sediment concentration $\mathrm{U}_{0}$ takes from $\mathrm{Uc}$ and $\mathrm{Ut}$, the vicinity of each of the two times has a the sediment concentration peak, which has little numerical difference. And this is quiet different from sediment concentration under the conditions of stocks wave. The latter only has a smaller peak moment in the vicinity of Ut only when very close proximity to bed. It is guessed that this situation is due to more sediment settling down in the times when velocity is small (with the wave propagation in opposite directions)because of the large difference between Uc and Ut under the condition of stocks wave in sample 2. Uc and Ut refer to the maximum flow speed towards and reverse the wave direction. 
Time-averaged sediment concentration is calculated by time averaging instantaneous values, which are calculated by averaging instantaneous rate of sediment in different ways that mentioned in the previous text. Measured data is taken from Ribberink and Al-Salem's experimental data, in the case of sample 2. Mainly calculates the situation near the bottom of the bed within $6 \mathrm{~cm}$. Figure 7 . shows the results.

The "You formula" in the chart means the curve of time-averaged sediment concentration using the method putting the time-invariant eddy viscosity coefficient of the model such as You Zaijin into the formula (12). "Instantaneous value" means curve of time-averaged sediment concentration obtained by calculation instantaneous coefficient of eddy viscosity into the formula (12) in the flow speed calculation. According to the diagram, the two curves closer to the calculation when near to the bed (less than $1 \mathrm{~cm}$ ), and the trend of separation increases when the distance increases. Compared with the measured data, the two calculated curves correspond well in near-bottom $(2 \mathrm{~cm})$. The farther away from the bed, the smaller values were got. Figure 7. reflects a very good simulation result of the calculation value of sediment concentration in the entire space. If only time-invariant sediment concentration is needed, eddy viscosity coefficient calculation is adequate. In this way we can greatly simplify the calculation. Therefore, in the general engineering calculations, the sediment diffusion coefficient is usually taken as a constant or a function (Wang Huizhong 2001, Koutitas 1987) changes with the water depth.

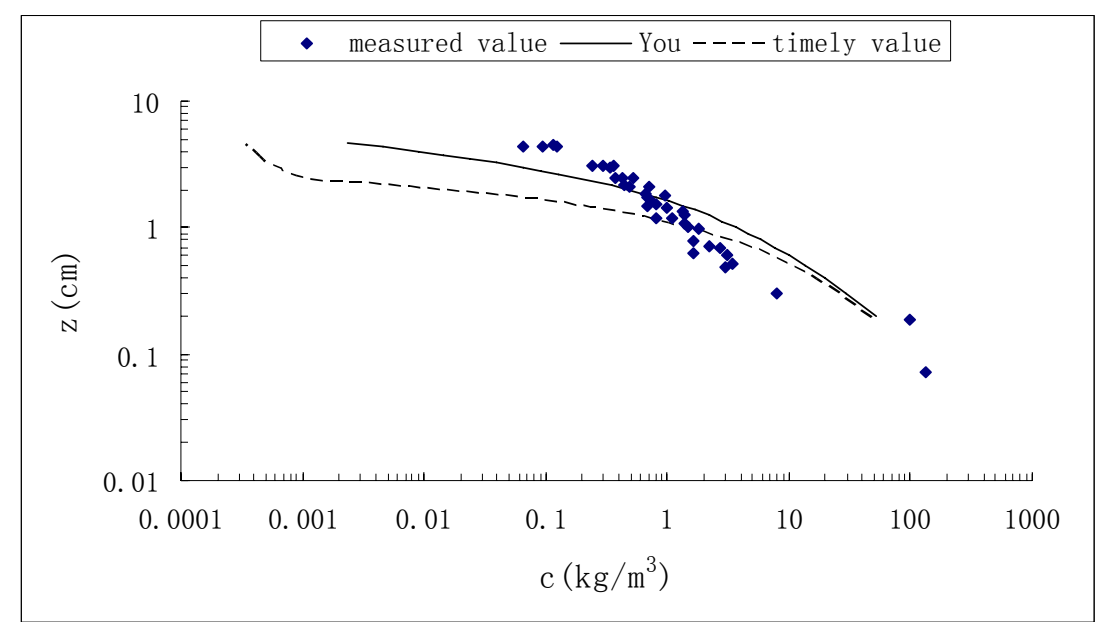

Figure 7. Vertical distribution of time-averaged sediment concentration

\section{CONCLUSIONS}

Simulating sediment concentration in wave boundary layer, two of the most important aspects are getting reasonable values of the vertical and sediment of the reference sediment concentration at the bottom. It is generally believed that the instantaneous values calculated certainly better than the use of time-invariant model of the diffusion coefficient, the calculation of model results in this article also proved this point.

According to the method in this article which is associating vertical eddy diffusion coefficient with viscosity coefficient, using turbulence model eddy viscosity calculated by the instantaneous values is better than use of time-invariant model, the method is feasible, and it is considered to get a better viscosity model which could obtain numerical coefficient, in order to obtain better simulation results. As for the reference sediment concentration, the approach can be used in a certain degree of effective simulation, but simulation results show that under the conditions of stocks wave it is not very satisfactory, and it is need for further experimental and theoretical studies to obtain a more reasonable conditions of sediment concentration at the bottom.

\section{ACKNOWLEDGEMENTS}

This research was financially supported by "Commonweal Program of Chinese Ministry of Water Resources” (Project Number 200701026, 200901033). 


\section{REFERENCES}

A.A.Al-Salem. 1993. Sediment transport in oscillatory boundary layers under sheet-flow conditions.

Jan S. Ribberink and Abdullah A. Al-Salem. 1995. Sheet-flow and suspension of sand in oscillatory boundary layers, Coastal Engineering, 25, 205-225.

Jin Zhongqin. 1989. Numerical solution to the Navier and Stocks equations and turbulent models. Hohai university publishing company, Nan Jing.

Jorgen Fredsoe and Rolf Deigaard. 1992. Mechanics of coastal sediment transpor, World Scientific.

Koutitas C. 1987. Three dimension models of coastal circulation, an engineering viewpoint. Three dimensional Coastal Ocean Models, AGU. Washington D C.

Kang Suhai, Yang Yihang. 1995. An application of k- $\varepsilon$ model in turbulent bottom boundary layers in water waves. Journal of Hydrodynamics, 10, No.5.

Nielson P. 1992. Coastal bottom boundary layers and Sediment transpor. World Scientific.

Qian Ning, Wan Chaohui. 1983. Mechanics of Sediment Transport. Science Press, Beijing.

Tao Wenquan. 2001. Numerical heat transfer. Xi'An Jiao Tong University Publishing Company.

Wang Deguan. 1989. Computational Hydrodynamics theory and application. Hohai University Publishing Company.

Wang Huizhong, Song Zhiyao and Xue Hongchao. 2001. A Quasi-3D numerical model of WindDriven current in Taihu Lake considering the variation of vertical coeff icient of Eddy Viscosity. Journal of lake Sciences, 13( 3).

Yan Bin, Zhang Qinhe. 2006. Vertical suspended sediment concentration due to waves. Journal of Sediment Research, 5.

Yang Yihang, Yang Xiuzhi. 1988. Theoretical and experimental investigation on the oscillatory boundary layer beneath water waves. Journal of Hydraulic engineering, 11.

Zhao Chongjiu. 1993. Laboratory study on the characteristics of fine sand bed under waves.

Z.J. You, D.L. 1992. Wilkinson and P. Nielson. Velocity distribution in turbulent oscillatory boundary layer. Coastal Engineering, 18, 21-38

Yuliang Zhu, Jing Ma and Peipei Dong. 2009. The improvement of k- $\varepsilon$ model in turbulent wave boundary layer model. $28^{\text {th }}$ OMAE, 2009 